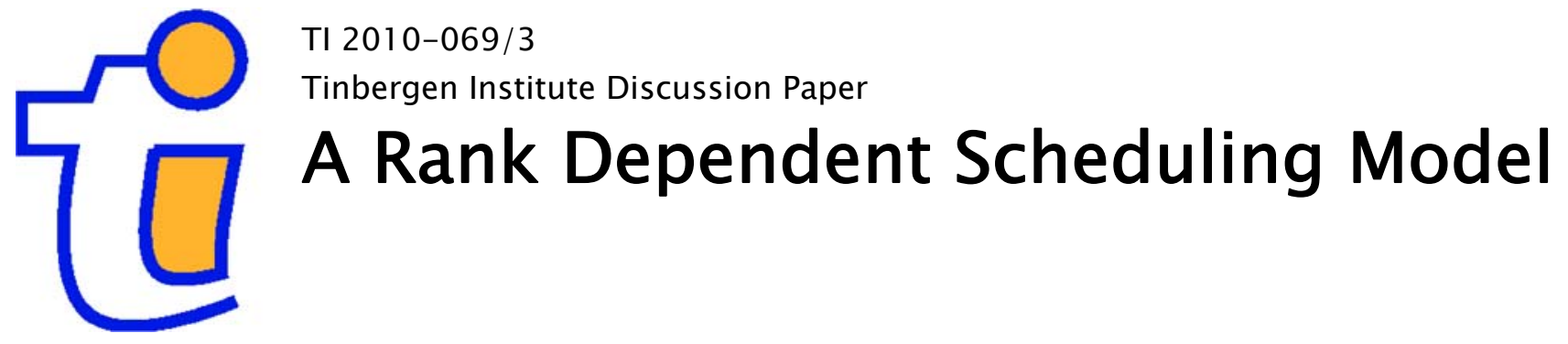

Paul Koster ${ }^{1}$

Erik T. Verhoef

I VU University Amsterdam;

2 Tinbergen Institute. 


\section{Tinbergen Institute}

The Tinbergen Institute is the institute for economic research of the Erasmus Universiteit Rotterdam, Universiteit van Amsterdam, and Vrije Universiteit Amsterdam.

Tinbergen Institute Amsterdam

Roetersstraat 31

1018 WB Amsterdam

The Netherlands

Tel.: +31(0)205513500

Fax: $+31(0) 205513555$

Tinbergen Institute Rotterdam

Burg. Oudlaan 50

3062 PA Rotterdam

The Netherlands

Tel.: + $31(0) 104088900$

Fax: $+31(0) 104089031$

Most TI discussion papers can be downloaded at http://www.tinbergen.nl. 


\title{
A rank dependent scheduling model
}

\author{
Paul Koster ${ }^{1}$ and Erik T. Verhoef ${ }^{1,2}$ \\ 1 Department of Spatial Economics, VU University Amsterdam, The Netherlands \\ 2 Tinbergen Institute, The Netherlands
}

Address for correspondence: VU University Amsterdam, Faculty of Economics and Business Administration, Department of Spatial Economics, De Boelelaan 1105, 1081HV Amsterdam, The Netherlands.

\section{Acknowledgements}

We like to thank Peter Wakker, participants of ETC 2009, and an anonymous reviewer for valuable comments on earlier versions of this paper. Furthermore, we like to thank Jasper Knockaert for provision of the data. The usual disclaimer applies. The data were gathered in the context of the Dutch peak avoidance project 'Spitsmijden'. Paul Koster was partly funded by TRANSUMO. The project "Reliable accessibility of airports" was made possible with support of TRANSUMO (TRANsition SUstainable MObility). TRANSUMO is a Dutch platform for companies, governments and knowledge institutes that cooperate in the development of knowledge with regard to sustainable mobility.

\begin{abstract}
This paper proposes an analytical framework for scheduling decisions of road travelers that takes into account probability weighting using rank dependent utility theory. The fundamental difference with the standard scheduling model based on expected utility is that the probabilities of arrivals are treated in a non-linear way. This paper shows how scheduling decisions are affected by the weighted probabilities of the traveler. We derive the costs of non-optimal chosen departure times because of probability weighting and show that if the parameterized probability weighting function is similar to what has been found for gambling, the costs of probability weighting for morning peak car travelers are around 3 per cent. For the full range of parameters tested, we find costs in the range of 0-24 percent of total travel costs.
\end{abstract}

Date of final version: $26 / 08 / 2010$

Corresponding author: Paul Koster. Tel.: +31 2059 83964. Fax.: +31 205986004.

Email addresses: pkoster@feweb.vu.nl (P.R. Koster), everhoef@ feweb.vu.nl (E.T. Verhoef). Address: Faculty of Economics and Business Administration. De Boelelaan 1105, 1081HV Amsterdam, The Netherlands. 


\subsection{Introduction}

The last decade researchers and policy makers have paid considerable attention to user benefits from an increased reliability of transport systems. Stated preference and revealed preference estimations show that travelers are willing to pay money to avoid travel time variability caused by unreliable transport systems (for overviews: RAND Europe, 2004; Brownstone and Small, 2005; Tseng, 2008; Li et al., 2010). Early research of Gaver (1968) and Knight (1974) already revealed the intuitive mechanism that an increase in the standard deviation of travel time leads to earlier departure times and corresponding higher travel costs. Our model uses this intuition and builds on the work of Small (1982) and Noland and Small (1995), that uses the concept of schedule delay to analyze the costs of travel time variability. In this view, travelers are not so much concerned by statistical measures as the standard deviation, but dislike travel time variability primarily because they can arrive early or late. They, of course, to some extent can anticipate on variable travel times by choosing their departure time optimally.

In the model of Noland and Small (1995), the natural assumption was made that travelers treat probabilities in an essentially linear way; travelers treat a probability that is twice as high as twice as likely. From the behavioural economic literature there is however quite some evidence that this is not the case in practice, and that probabilities are weighted in a non-linear way (Wakker, 2010). Some recent empirical evidence shows that this may also be true for travel decisions (Hensher and Li, 2010).

In this paper, we show how such probability weighting affects the choice of departure time, and how the travel costs are affected by probability weighting. The paper is organized as follows: in the next section we show the relationship of our model with earlier literature. In section 3 we introduce probability weighting. In section 4 we present the behavioural model. 
In section 5 this model is applied using camera data from a highway in The Netherlands.

Section 6 concludes and gives directions for future research.

\subsection{Literature}

The scheduling model of Small (1982) has become the workhorse model for evaluating the costs of travel time variability. ${ }^{3}$ The model is based on earlier work of Vickrey (1969) and shows how departure time decisions affect travel costs and how travelers choose their departure time $\left(t_{h}\right)$ given their preferred arrival time $\left(t_{p a t}\right)$. The central idea is that travelers make a trade-off between travel time costs, and costs of being early or late. In the simplest setting, the cost function of a traveler with departure time from home $t_{h}$ is linear in its arguments and is given by equation (1), where the headstart $H$ is defined as $t_{p a t}-t_{h}$ and $T$ as the total travel time. A discrete penalty for lateness, originally present in Small's model, is not included to keep the model simple and because it is usually found to be insignificant, at least in Dutch applied research (see for example: Tseng, 2008). Other costs, such as fuel costs, are ignored also for simplicity. We than have:

$$
\begin{gathered}
C[H]=\alpha \cdot T+\beta \cdot S D E+\gamma \cdot S D L \\
\text { with: } \\
S D E=\max (0, H-T) \\
S D L=\max (0, T-H)
\end{gathered}
$$

In equation (1) the amount of time being early, or schedule delay early, is given by $S D E$ and schedule delay late is given by $S D L$. The value of travel time (VOTT) is given by $\alpha$, the value of schedule delay early (VSDE) by $\beta$, and the value of scheduling delay late (VSDL) by $\gamma$.

\footnotetext{
${ }^{3}$ In the literature about travel time variability the definition of travel time variability is frequently referred to as uncertainty or reliability. We define uncertainty as not knowing the probability distribution and variability as the variation of travel times. Reliability can be used for the performance of the transport system.
} 
These values have been frequently estimated in the literature. ${ }^{4}$ Empirical work shows that usually the relation $\beta<\alpha<\gamma$ holds.

This paper is not concerned with the estimation of the WTP coefficients of equation 1 . The main focus in this paper will be how the departure time choice of a traveler is affected by these parameters and by the travel time when it is stochastic.

The original model of Small (1982) was extended to the case of stochastic travel times by Noland and Small (1995). They assume that the cost function is linear in its arguments and define the expected costs as in equation (2), where travel times are distributed with a probability density function $f(T)$.

$$
\begin{gathered}
E(T)=\int_{0}^{\infty} T \cdot f(T) d T \\
E(S D E)=\int_{0}^{H}(H-T) \cdot f(T) d T \\
E(S D L)=\int_{H}^{\infty}(T-H) \cdot f(T) d T \\
E(C[H])=\alpha \cdot E(T)+\beta \cdot E(S D E)+\gamma \cdot E(S D L)
\end{gathered}
$$

Trip time decisions in this model are thus analyzed in an expected costs framework where it is assumed that the travel costs are linear in its arguments. Travelers determine their optimal headstart given $f(T), t_{p a t}$ and $\alpha, \beta$ and $\gamma$. For the exponential and the uniform distribution Noland and Small (1995) showed the relationship between the optimal total expected costs and the distribution parameters. Later research analyzed the model for a time dependent lognormal, Weibull and gamma travel time distribution (Koster et al., 2009) and for a general travel time distribution (Fosgerau and Karlström, 2010). The main motivation for these extensions was given by the fact that the parameters of the travel time distribution are not constant over time-of-day, and that the distribution of travel time is skewed (van Lint et al., 2008). A particularly nice result of Fosgerau and Karlström (2010) is that the expected costs

\footnotetext{
${ }^{4}$ For overviews of empirical studies we refer to Brownstone and Small (2005), Tseng (2008) and Li et al. (2010).
} 
of a traveler who chooses his optimal departure time are linear in the standard deviation of travel times if it is assumed that the standardized distribution is independent of the departure time.

A critical assumption of the Noland and Small model is that travelers know the distribution of travel time, and treat probabilities in a linear way, meaning that the ratio of weights attached to different outcomes is equal to the ratio of the probabilities. Because the model is usually applied for calculating the costs of commuting, the main argument for the first assumption is that travelers learn from earlier experiences. Ettema and Timmermans (2006) noted that the assumption of perfect knowledge can be unrealistic, and they therefore introduce the concept of a subjective probability distribution to analyze the potential benefits of travel information. In their model they assumed that travel information will result in a better perception of the probability distribution, and therefore in lower travel costs. A major drawback of their analysis is that the relationship between the subjective and the objective probabilities is not defined explicitly. Therefore changes in the parameters of the objective distribution cannot be analyzed as long as the relationship with the subjective distribution is not known, and cost-benefit analysis is not possible. Furthermore they assume that the subjective probabilities are treated in a linear way.

The second assumption of the Noland and Small (1995) model is that travelers treat probabilities in a linear way, so perceived probabilities are not affected by the risk attitude of the traveler. Batley (2007) analyzes scheduling decisions using prospect theory with a discrete representation of departure times. He uses a transformation of the utility value of a prospect to analyze the effect of variable travel times when travelers are risk averse or risk seeking. The risk attitude is his model is then captured by the curvature of the utility function. Our approach differs from the approach of Batley (2007) in that we transform the probabilities, instead of the utility values of the arrivals. The idea of transforming the probabilities goes 
back to the work of Preston and Baratta (1948) and Mosteller and Nogee (1951). This approach is intuitive, since the risk perception of travelers is likely to primarily affect the perceived probabilities rather than the utility function. Recent empirical work by Hensher and $\mathrm{Li}$ (2010) in the context of travelers' decisions, suggests that the risk perception would affect both perceived probabilities and the utility function. This extension could be made to our model, but for now we choose to focus on probability weighting.

The risk perception of travelers is affected by at least two factors. The first factor is how travelers understand the concept of probability. It could be that travelers cannot make a distinction between different outcomes, and for example simply treat all outcomes as equally likely. The second factor is how pessimistic or optimistic travelers are. Pessimistic travelers will pay more attention to bad outcomes and therefore they assign a higher weight to these outcomes (Wakker, 2010). In this paper, rank dependent utility theory is used to analyze departure time decisions when probabilities are weighted. The intuition behind rank dependence is that the attention that is given to a certain outcome does not only depend on the probability of that outcome, but also on the ranking of the outcomes. We use a probability weighting function for a general cumulative travel time distribution. This weighting function transforms the probabilities into decision weights (Diecidue and Wakker, 2001).

This paper makes two contributions to the literature. First, we show analytically how probability weighting affects departure time decisions of travelers for a time-of-day independent travel time distribution. We do this to show the basic intuition of the effect of probability weighting on departure time choice. Second, the rank dependent scheduling model is formulated for a time-of-day dependent travel time distribution and is compared to the standard scheduling model, to analyze how large the effect of probability weighting is on expected travel costs. If the effect is not large, policy makers can ignore probability weighting and use the simpler expected costs model to analyze the effect of travel time variability on the 
behaviour of travelers. This will be less costly to analyze, since there is no need to measure the probability weighting functions of individual travelers.

\subsection{Rank dependent utility}

This section introduces the concept of rank dependent utility which can explain the violations of behaviour consistent with expected utility as revealed by Allais (1953). Allais (1953) showed that decision makers transform probabilities when they face a risky choice, and that they do not treat probabilities linearly. This gave rise to the development of new behavioural theories that could explain why expected utility maximization is violated.

A central element in rank dependent utility models is the probability weighting function, which defines a relationship between the cumulative density function $(C D F)$ and the weighted $C D F$. In our context, this weighted $C D F$ is used by the traveler to determine the optimal departure time from home. When a continuous representation of probability is used, a probability weighting function is needed that can describe a transformation of the cumulative density function of the travel times $F(T)$. The weighting of the $C D F$, rather than weighting the probabilities themselves, is central in rank dependent utility theory and is based on the work of Quiggin (1982) and Schmeidler (1986). They extend the earlier theory of Kahneman and Tversky (1979) where the probabilities of the probability density function $(P D F)$ were weighted. This model leads to problems since stochastic dominance may be violated. ${ }^{5}$ Quiggin (1982) analyzed the case where the cumulative probabilities of events were known and transformed by a probability weighting function. These weighted cumulative probabilities are called decision weights. Schmeidler (1986) analyzed the case where the probabilities were unknown. In his model he proposed event-decision weights because the weights are based on the ordering of the events.

\footnotetext{
${ }^{5}$ If the weighting function of the $P D F$ is nonlinear, there is the possibility of an increasing utility but a lower evaluation value of an outcome (Wakker, 2010).
} 
Kahneman and Tversky (1992) based their model - which is well known as cumulative prospect theory $(C P T)$, as opposed to "original prospect theory" of Kahneman and Tversky (1979) - on the work of Quiggin (1982) and Schmeidler (1986). The difference between $C P T$ and rank dependent utility is that $C P T$ is able to account for loss aversion and reference dependence. This means that travelers evaluate outcomes as gains or losses compared to a reference point. Loss aversion is measured through a utility function which is kinked at the reference point and is ignored in this paper since it is difficult to determine in this context whether there exists a clearly defined reference point of the traveler, and if so, what it is. $C P T$ also uses separate probability weighting functions for the loss and the gain domain. For example, De Borger and Fosgerau (2008) estimate loss aversion in a study on the VOTT. An intuitive concept could be to weight the probabilities for early and late arrivals separately, or to use the reference arrival of $t_{h}+E\left(T\left[t_{h}\right]\right)$. However, it is not clear if the travelers use the mean or the mode (or another measure) as their reference point.

The shape of the weighting function can be explained by two behavioural factors. First, travelers can be 'likelihood insensitive'. Likelihood insensitivity means that travelers do not understand the concept of probability well. There are two types of likelihood insensitivity, which we can picture by imagining a graph with the $C D F$ on the horizontal axis and the weighted $C D F$ on the vertical axis. A frequently found weighting function is the inversely Sshaped which is relatively horizontal in the middle and steep at both ends, meaning that travelers overweight extreme outcomes (Tversky and Wakker, 1995). Another possibility is the S-shaped weighting function, where in the extreme case travelers entirely ignore the variability of travel times and treat the travel distribution as if it has 1 possible outcome.

Second, travelers can be pessimistic (risk averse) or optimistic (risk seeking). In the rank dependent scheduling model this risk attitude is modeled with the weighting function instead of the utility function. The three typical cases of pessimism (risk aversion), optimism 
(risk seeking) and likelihood insensitivity are given in figure 1 (Wakker, 2010). Here we assume that the outcomes are ranked from good to bad.

<<insert Figure 1 about here〉>

The probabilities are weighted according to equation (3), where $F(T)$ is the measured $C D F$ and $W[F(T)]$ the weighted $C D F$.

$$
w[F(T)]=\frac{\partial W[F(T)]}{\partial T}=\frac{\partial W[F(T)]}{\partial F(T)} \cdot f(T)
$$

The probabilities of the measured $P D F$ are given by $f(T)$, and are weighted by the first derivative of the weighted $C D F$ with respect to the cumulative density function $F(T)$ to obtain the weights. In the next section it is analyzed how probability weighting affects the choice of departure times.

\subsection{Optimal choice of departure time}

\subsection{Ranking of the outcomes}

In this section we discuss the ranking of the outcomes in terms of travel costs. We assume that the preferences are bundled, meaning that we do not use different probability weighting functions for $T, S D E$ and $S D L$. Therefore, the decision weights are applied to the full outcome, i.e. the full set of attributes jointly, and not to individual attributes separately. For late arrivals, it is clear that a longer travel time implies, for a given departure time, a higher travel cost: both travel delay and schedule delay costs would increase. But when we make the conventional assumption that $\beta<\alpha$, a longer travel time also implies higher travel costs for early arrivals (again given the moment of departure). This assumption, $\beta<\alpha$, is rather intuitive, as it boils down to assuming that an early arriving traveler prefers terminating the trip above making a detour and benefiting at a rate $(\beta-\alpha)$ from such a voluntary trip duration extension. 
Bundling is therefore justified in our context, implying that travelers can be assumed to rank the possible outcomes for a given departure time according to travel times. Our approach thus differs from the one proposed by Hensher and $\mathrm{Li}$ (2010), where travel times are ranked in terms of late arrival (least attractive), on-time arrival (most attractive) and early arrivals (in between). Their approach is not applicable in our setup, in which also the size of the schedule delay is important in determining the rank of the outcome, and not only the fact whether a traveler arrives early or late.

We rank the travel times from good to bad, which results in a travel cost ranking from low to high. The rank dependent travel costs are given by equation 4, where the travel time distribution is dependent on $H$. For simplicity we assume that the unit WTP values $(\alpha, \beta, \gamma)$ are independent of the time of day (see Tseng and Verhoef, 2008, for further discussion).

$$
R D(C[H])=\int_{0}^{\infty} C[H] \cdot \frac{\partial W[F[T ; H]]}{\partial F[T ; H]} \cdot f[T ; H] d T
$$

Inserting the cost function of equation 1 in equation 4, this can be rewritten as:

$$
\begin{aligned}
& R D(C[H])=\alpha \cdot \mu_{w}[H]+\beta \cdot \int_{0}^{H}(H-T) \cdot \frac{\partial W[F[T ; H]]}{\partial F[T ; H]} f[T ; H] d T+\gamma \cdot \int_{H}^{\infty}(T-H) \cdot \\
& \frac{\partial W[F[T ; H]]}{\partial F[T ; H]} f[T ; H] d T
\end{aligned}
$$

In equation $5, \mu_{w}[H]$ is the weighted mean travel time which depends on the departure time from home and therefore on the headstart $H$, because the travel time distribution depends on $H$. This rank dependent cost function will be used in the next sections to determine the optimal headstart and the numerical analysis.

\subsection{Optimal choice of headstart}

In this section the optimal headstart for a traveler is determined for a time-of-day independent travel time distribution, so we assume $F[T ; H]=F[T]$. Our analysis in this section follows Fosgerau and Karlström (2010). This section is mainly to show the intuitive effects of changes 
in the probability weighting function on the choice of departure time. We standardize the travel time distribution such that $T=\mu+\sigma x$, where $\mu$ is the mean, $\sigma$ the standard deviation of travel times and $x$ is a stochastic variable distributed with a cumulative distribution function $G[x]$. Fosgerau and Karlström (2010) showed that in this case the first derivative of the expected cost function of equation 2 is given by:

$$
\frac{\partial E(C[H])}{\partial H}=-\gamma+(\beta+\gamma) \cdot G\left[\frac{H-\mu}{\sigma}\right]
$$

The solution for the optimal headstart can be found by setting this first order condition to 0 , and solve for $H$. The optimal headstart is given in equation 7 and is linear in the standard deviation of travel times.

$$
H F^{*}=\mu+\sigma \cdot G^{-1}\left[\frac{\gamma}{\beta+\gamma}\right]
$$

This solution holds for a general distribution and is unique because the cost function is convex for all values of $H .^{6}$ Now assume that a traveler chooses the optimal $H$ according to the weighted cumulative distribution function $W[G[x]]$. The first-order condition of equation 6 changes into:

$$
\frac{\partial R D(H)}{\partial H}=-\gamma+(\beta+\gamma) \cdot W\left[G\left[\frac{H-\mu}{\sigma}\right]\right]
$$

The solution for the optimal headstart when probabilities are weighted is given by equation (9) where the inverse of $W$ is taken with respect to $G[x]{ }^{7}$

$$
H W^{*}=\mu+\sigma \cdot G^{-1}\left[W^{-1}\left[\frac{\gamma}{\beta+\gamma}\right]\right]
$$

Again the optimal headstart is linear in the standard deviation of travel times and the solution is unique because the rank dependent cost function is convex in $H$. The next figure shows the implication of this result.

\footnotetext{
${ }^{6}$ As long as $\mathrm{G}^{\prime}[\mathrm{x}]>0$ the solution is unique. Note that the second derivative of the expected cost function is given by: $\mathrm{G}^{\prime}[(H-\mu) / \sigma] \cdot(\beta+\gamma) / \sigma>0$.

${ }^{7}$ Suppose we want to solve $W[G[x]]=z$ for $x$. First substitute $y=G[x]$, so $W[y]=z$. Solving for $y$ using the inverse rule gives the solution $y=W^{1}[z]$ which implies $G[x]=W^{1}[z]$ if we substitute back $y=G[x]$.Applying the inverse rule again for $\mathrm{x}$ gives $x=G^{-1}\left[W^{1}[z]\right]$. The solution is unique because $W[G[x]]$ is a strictly increasing function in $G[x]$, and $G[x]$ is a strictly increasing function $x$.
} 
<<insert Figure 2 about here>>

In figure 2 the optimal headstart for the standard scheduling model is given by $H F$. First, assume that a traveler is optimistic. This means that probabilities of low travel costs are overweighted and probabilities of high travel costs are underweighted. The weighting function is given by $W 1$ in figure 2 . This weighting function is always above the $G[x]$ function (except for the corners). This means that the solution for the optimal headstart which is given by $H I$ in the figure - is always smaller than $H F$. If travelers are pessimistic, the weighting function is given by $W 2$ and is always below $G[x]$. In that case the optimal headstart is always larger than $H F$.

The effects of optimism and pessimism on the travel costs have been analyzed by Koster (2009). Optimistic travelers will arrive late more frequently, and pessimistic travelers arrive early more frequently. In the empirical application, he finds that it is more costly to be an optimistic than a pessimistic traveler.

<<insert Figure 3 about here>>

The case of likelihood insensitivity is given in figure 3 , where the weighting function is inversely S-shaped. The effect on the optimal headstart $(H 3)$ depends on the value $\gamma /(\beta+\gamma)$. If we define $c^{*}$ as the intersection point of $W 3[$.$] and G[.], the optimal headstart is lower than$ $H F$ for $\gamma /(\beta+\gamma)<c^{*}$, and higher than $H F$ for $\gamma /(\beta+\gamma)>c^{*}$. If $\gamma /(\beta+\gamma)=c^{*}$, travelers choose the optimal headstart and the costs of probability weighting are 0.

\subsection{Extension to a model with a time-of-day dependent travel time distribution}

In this section we formulate the model for a time-of-day dependent travel time distribution. It is assumed that the traveler optimizes $H$ given his rank dependent cost function of equation 5 . 
Denote the optimal headstart with probability weighting by $H W$, and without probability weighting (so when $W[F[T ; H]]=F[T ; H]$ ) by $H F$. The costs of probability weighting $(C O P W)$ are given by equation (10) and are equal to the extra costs because of probability weighting, compared to the expected costs model.

$$
C O P W=E(C[H F])-E(C[H W])
$$

The $C O P W$ are always $\geq 0$ because the expected costs are calculated on the basis of objectively expected costs. Travelers never can do better than the expected costs model. As already shown by Fosgerau and Karlström (2010), there is no closed-form solution available if the travel time distribution depends on $H$. Therefore we will use numerical examples in the next section to calculate the costs of probability weighting.

\subsection{Empirical application}

In this section we will analyze, for a numerical example, the effects of likelihood insensitivity, and of optimism and pessimism,. From a policy perspective it is useful to consider both phenomena, since the type of information that will improve the departure time decision will differ. If travelers are likelihood insensitive they need more information about how to deal with probabilities, how to understand differences between probabilities, and possibly feedback on their decisions can help to improve these on future occasions (see for example Van de Kuilen 2009 for a recent empirical test). If travelers are optimistic or pessimistic, more experience in travelling or more information on expected values can help to obtain a more appropriate view of the travel time distribution. In our numerical analysis we will use the weighting function of Prelec (1998) given in equation (11):

$$
W_{\text {Prelec }}[F[T]]=e^{-\eta \cdot(-\ln F[T])^{\theta}}, \theta>0, \eta>0
$$

The weighted $C D F$ of equation (11) is not defined at the point $F[T]=0$, but in the limit

$W_{\text {Prelec }}$ will go to 0 if $F(T)$ goes to 0 and it equals 1 if $F(T)$ equals 1 . The first derivative with 
respect to $T$ is always larger than 0 as long as $\theta>0$. When $\eta=\theta=1$, equation (11) reduces to $F(T)$. When the parameter $\eta$ increases, the weighting function shifts down and travelers are more pessimistic: they will overweigh the probabilities of bad outcomes, so high travel costs. The parameter $\theta$ controls the shape of the weighting function. When $\theta$ goes to 0 , the weighting function will be extremely inverse S-shaped. This means that travelers treat the distribution as if it has two mass points at the extremes. If $\theta$ goes to infinity, the distribution collapses into one intermediate mass point and the weighting function will be extremely Sshaped.

The travel time data we use has been obtained using license plate detection for a highway road stretch between Gouda and Zoetermeer, located near The Hague in the dense south-western part of the Netherlands, between March 2008 and July 2009. Only workdays are included, and school holidays are omitted. The free-flow travel time is around 5 minutes. The individual car data is aggregated to 5 minute time-of-day intervals. For every day the median travel time of an interval is used as a travel time observation. The median is used instead of the mean, because the mean is more influenced by outliers caused by the fact that there are ramps along the link, allowing some travelers to temporarily leave the road between the two points of measurement. The final dataset is interpolated to obtain 1 minute interval data. A travel time distribution has been fitted for every time period using a kernel smooth density estimator. ${ }^{8}$ Therefore, no distributional assumptions are needed. ${ }^{9}$ The time-of-day dependent mean and variance are plotted in figure 4. <<insert figure 4 about here>>

\footnotetext{
${ }^{8}$ For each time period, we fit the travel time distribution using an optimal data-driven bandwidth, and use 100 equally spaced points (Bowman and Azzalini, 1997). All programming has been done in Matlab 7.6.0. The programming code is available on request.

${ }^{9}$ For example, Noland and Small (1995) assumed that the delays are exponential or uniformly distributed. Koster et al. (2009) assumed that the delays are Weibull, gamma or lognormal distributed and Fosgerau and Karlström (2010) assumed that the standardized distribution of travel times does not change over day-time.
} 
We choose typical values for the model parameters which are based on earlier estimations for Dutch commuters: VOTT $=8 € / \mathrm{h}, \mathrm{VSDE}=0.6 * V O T T$ and $V S D L=1.4 * \mathrm{VOTT}$ (Tseng, 2008). For the parameters of equation (11) we choose the base values obtained from studies about gambling: $\theta=0.65$ and $\eta=1.1$ (Wakker, 2010), and will perform a sensitivity analysis afterwards. First, we show in figure 5 how the optimal headstart is affected by probability weighting. We do so by plotting the expected costs with and without probability weighting for an optimistic $(\eta=0.5)$ and a pessimistic $(\eta=1.5)$ traveler with $t_{\text {pat }}=8: 30$.

<<insert figure 5 about here>>

As expected, the rank dependent costs of the optimistic traveler is lower than without probability weighting, and the rank dependent costs for the pessimistic travelers is higher. The intuitive result of section 4.2 remains the same for a time-of-day dependent distribution. Optimistic travelers choose a smaller headstart than that without probability weighting, and pessimistic travelers choose a longer headstart. Note that the optimal headstarts are given by the global minima of the curves in figure 5 .

The resulting optimal expected costs for all preferred arrival times are plotted in figure 6. The travel time distribution is treated as given in our exercise, so no changes in equilibrium have been considered: the system is assumed to be in equilibrium already. <insert figure 6 about here>

The lowest line in figure 6 line gives the expected travel time costs without probability weighting. Those costs are proportional to the mean travel time. The middle line includes the expected scheduling costs. These scheduling costs are in the range of 10-31 per cent of the expected costs without probability weighting, and are somewhat higher than the empirical results of Fosgerau and Karlström (2010). The upper line indicates the expected travel costs when travelers apply probability weighting. The cost of probability weighting, $C O P W$, are 
between 0 and 8 per cent of the total travel costs, and the average $C O P W$ over the whole peak period are around 3 per cent of the total travel costs. The irregularities in travel costs with probability weighting can be explained by the fact that the distribution of travel times at different times of the day are estimated independently. ${ }^{10}$

Figure 7 shows the average share of the $C O P W$ in the total expected costs over the whole peak period for different parameter combinations of the weighting function. The COPW ranges from 0-24 per cent, and can be considered substantial when travelers are likelihood insensitive and pessimistic. The interpretation of the results is not straightforward because it is not possible to disentangle the effect of likelihood insensitivity and optimism/pessimism completely. For travelers that are not likelihood insensitive $(\theta=1)$, optimism $(\eta<1)$ is more costly than pessimism $(\eta>1)$. However the effect is rather small and the $C O P W$ are less than 3 per cent of total travel costs.

<insert figure 7 about here>

The effect of changes in the likelihood sensitivity parameter is higher. For values of $\theta<1$, higher pessimism is slightly more costly than optimism. For values of $\theta>1$, higher pessimism is approximately as costly as higher optimism. The combination of extreme pessimism and likelihood insensitivity results in the highest COPW (24.2 per cent). Empirical investigation and estimation of the probability weighting function must show what the appropriate values of $\theta$ and $\eta$ are that can be used in cost-benefit analysis.

Finally, we analyze the effect of different WTP values on the average share of the $C O P W$ in the total expected costs. Since only the relative values of VSDE and VSDL do matter for this Table 1, gives the results in terms of several values of VSDE and VSDL relative to VOTT, keeping $\eta$ and $\theta$ at the base values.

\footnotetext{
${ }^{10}$ We tested if for a given $t_{p a t}$ a traveler can have an earlier (or equal) expected arrival time with a later $t_{h}$. This is never the case, and therefore the expected arrival time is strictly monotonically increasing if $H$ decreases. Therefore the irregularities are not explained by the fact that there are few departure times with low expected costs.
} 
<insert Table 1 about here>

Table 1 shows that the average share of the $C O P W$ in the total travel costs is not so much changing for different WTP values and is in the range of 1.5-3.9 per cent.

\subsection{Conclusions}

In this paper, we developed a rank dependent scheduling model. Using the concept of probability weighting we are able to derive the costs of likelihood insensitivity, optimism and pessimism. If the parameterized probability weighting function for car travelers is similar to what has been found in the literature on gambling, then we find costs of probability weighting $(C O P W)$ for car travelers in the morning peak that are on average around 3 per cent. We show that this result is rather robust for different assumptions on the WTP values. This figure, however, naturally changes when the probability weighting function changes; for the ranges of parameters we tested, we found the $C O P W$ in the range of $0-24$ per cent.

The results must be interpreted with caution since there are very few studies in the area of travel behaviour that investigate the shape of the probability weighting function in the context of the scheduling model. The empirical estimation of probability weighting functions and the extension of the theoretical model using time-of-day dependent WTP values, nonlinear utility functions and loss aversion can be interesting directions for future research.

Another extension could address our assumption that the travel time distribution is exogenous. Therefore an interesting direction for future work can be to use an equilibrium model where the travel time distribution is determined by the number of travelers and the variation in road capacity. 


\section{References}

Bates, J., Polak, J., Jones, P., and A.J. Cook (2001): 'The valuation of reliability for personal travel', Transportation Research Part E, 37, 191-229.

Batley, R. (2007): 'Marginal valuations of travel time and scheduling, and the reliability premium', Transportation Research Part E, 43, 387-408.

Bowman, A. W. and A. Azzalini (1997): ‘Applied Smoothing Techniques for Data Analysis’, Oxford University Press, 1997.

Brownstone, D. and K.S. Small (2005): 'Valuing time and reliability: assessing the evidence from road pricing demonstrations', Transportation Research Part A, 39, 279-293.

De Borger, B. and M. Fosgerau (2008): 'The trade-off between money and travel time: A test of the theory of reference-dependent preferences', Journal of Urban Economics, 1, 101-115.

Diecidue, E., and P. Wakker (2001): ‘On the intuition of Rank-Dependent Utility', Journal of Risk and Uncertainty, 23, 281-298.

Ettema, D., and H. Timmermans (2006): 'Costs of travel time uncertainty and benefits of travel time information: Conceptual model and numerical examples', Transportation

Research Part C, 14, 335-350.

Fosgerau, M., and A. Karlström (2010): ‘The value of reliability', Transportation Research Part B, 44, 38-49.

Gaver, Jr. D.P. (1968): 'Headstart strategies to combat congestion', Transportation Science, 2, 172-181.

Hensher, D.A., and Z. Li (2010): 'Valuing Travel Time Variability within a Rank-Dependent Utility Framework and an Investigation of Taste Heterogeneity', 22 February 2010, ITLS, The University of Sydney.

Kahneman, D. and A. Tversky (1979): 'Prospect theory: An analysis of decision under risk.' Econometrica, 47, 2, 263-291. 
Kahneman, D. and A. Tversky (1992): 'Advances in prospect theory: Cumulative representation of uncertainty. Journal of Risk and Uncertainty, 5, 297-323.

Knight, T.E. (1974): 'An approach to the evaluation of changes in travel time unreliability: a “safety margin” hypothesis', Transportation Science, 3, 393-408.

Koster, P.R. (2009): 'The costs of uncertain travel times for car travelers'. Paper presented at the European Transport Conference 2009.

Koster, P.R., Verhoef, E.T., and E. Kroes (2009): 'The costs of variable travel times', Paper presented at Kuhmo Nectar Conference 2009.

Li, Z., Hensher, D.A., and J. Rose (2010): 'Willingness to pay for travel time reliability in passenger transport: A review and some new empirical evidence', Transportation Research E 46, 384-403.

Mosteller, F. and P. Nogee (1951): 'An experimental measurement of utility', Journal of Political Economy, 59, 371-404.

Prelec, D. (1998): ‘The probability weighting function', Econometrica, 66, 497-527.

Preston, M. G., and Baratta, P. (1948): 'An Experimental Study of the Auction Value of an Uncertain Outcome', American Journal of Psychology, 61, 183-193.

Quiggin, J. (1982): 'A Theory of Anticipated Utility', Journal of Economic Behaviour and Organization, 3, 225-243.

RAND Europe (2004): 'The valuation of quality and reliability in passenger and freight transport', AVV/RAND Europe, Rotterdam.

Schmeidler, D. (1986): 'Integral Representation without Additivity', Proceedings of the American Mathematical Society, 97, 255-261.

Small, K.A. (1982): 'The scheduling of consumer activities: work trips', American Economic Review, 72, 469-479. 
Tseng, Y.-Y. (2008): 'Valuation of Travel Time Reliability in Passenger Transport', PhD

Thesis, VU University Amsterdam.

Tseng, Y.-Y., and E.T. Verhoef (2008): 'Value of time by time of day: A stated preference study', Transportation Research B, 42, 607-618.

Tversky, A and P. Wakker (1995): 'Risk Attitudes and Decision Weights', Econometrica, 63, 1255-1280.

Van de Kuilen, G. (2009): 'Subjective Probability Weighting and the Discovered Preference Hypothesis', Theory and Decision, 67,1-22 .

Van Lint, J.W.C., Van Zuylen, H.J., and H. Tu (2008): 'Travel time unreliability on freeways:

Why measures based on variance tell only half the story', Transportation Research Part A, $42,258-277$.

Wakker, P. (2010): 'Prospect theory for risk and ambiguity', Cambridge University Press, forthcoming. 
Figure 1: Examples of pessimism or risk aversion (left), optimism or risk seeking (middle) and S-shaped likelihood insensitivity (right), when outcomes are ranked from best (left) to worst (right).
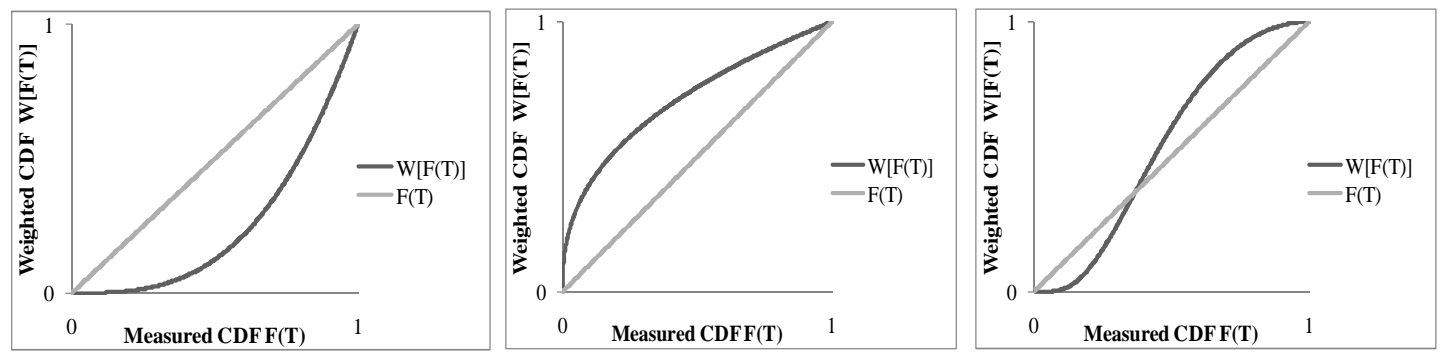
Figure 2: Optimized choice of headstart for optimistic (W1) and pessimistic (W2) travelers.

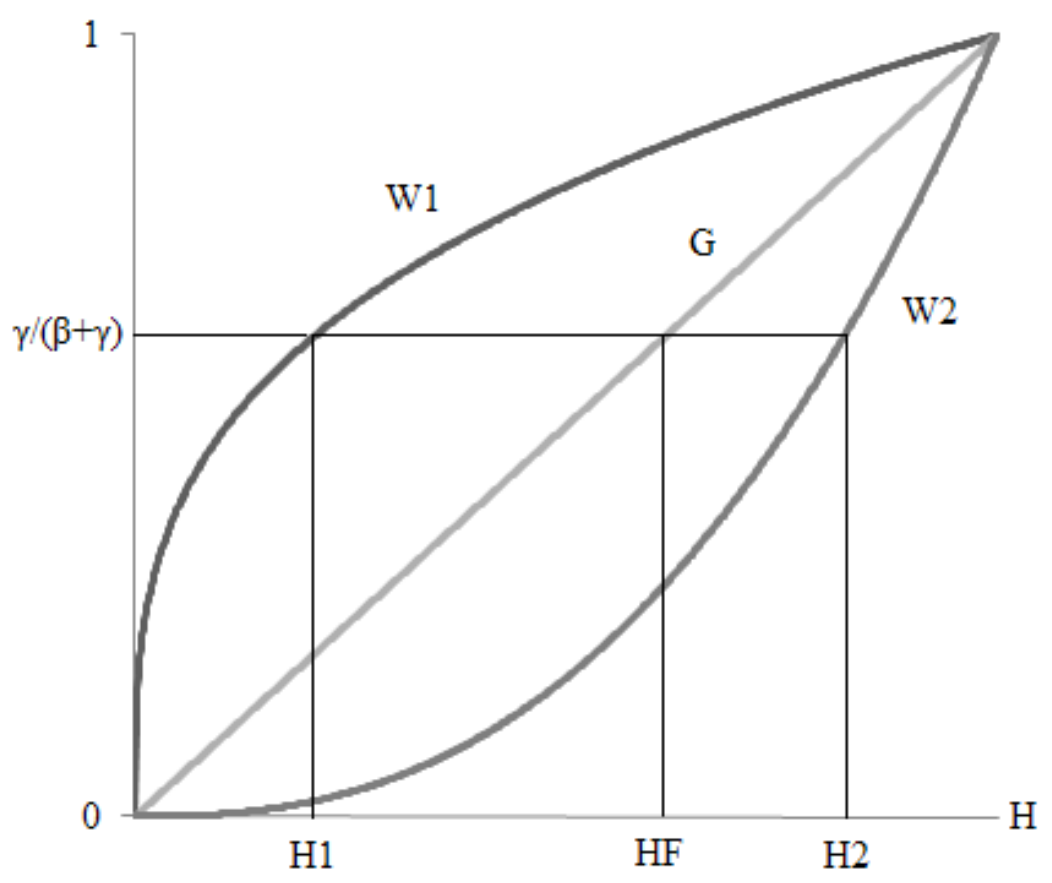


Figure 3: Optimized choice of headstart with likelihood insensitivity

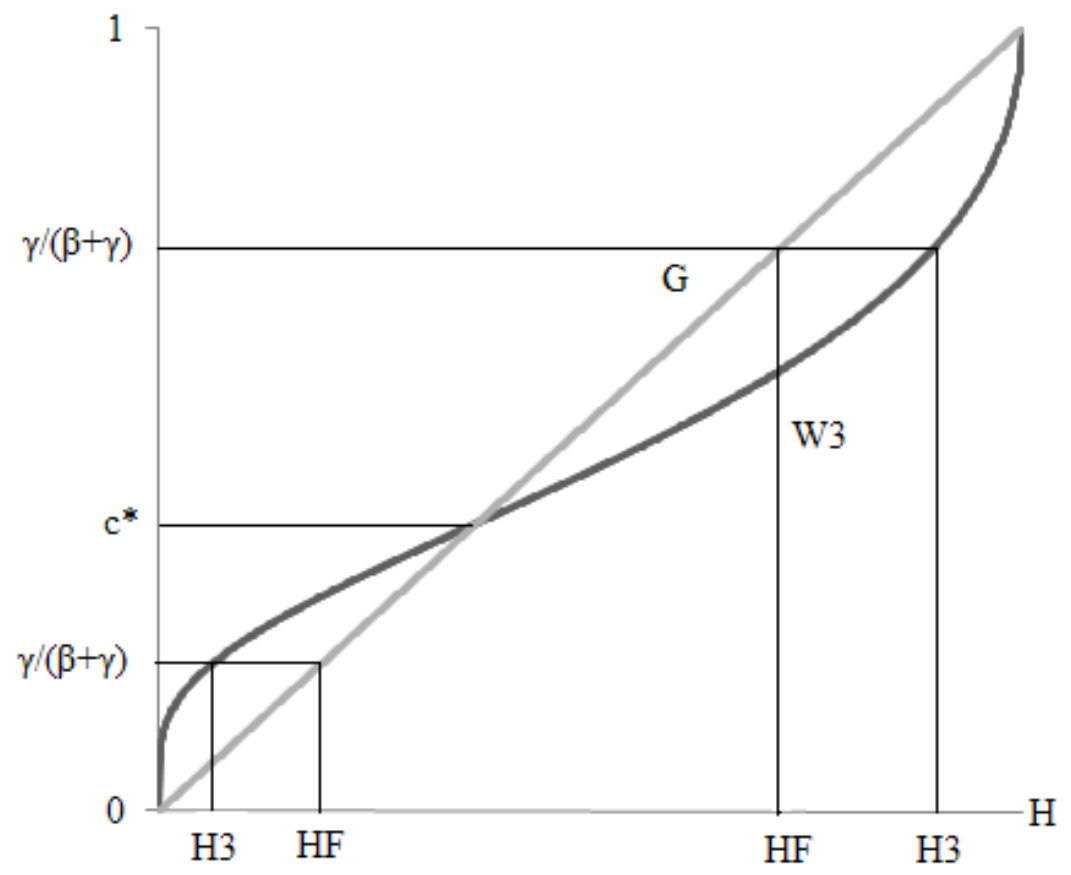


Figure 4: Time-of-day dependent mean and standard deviation of the observed travel time distribution.

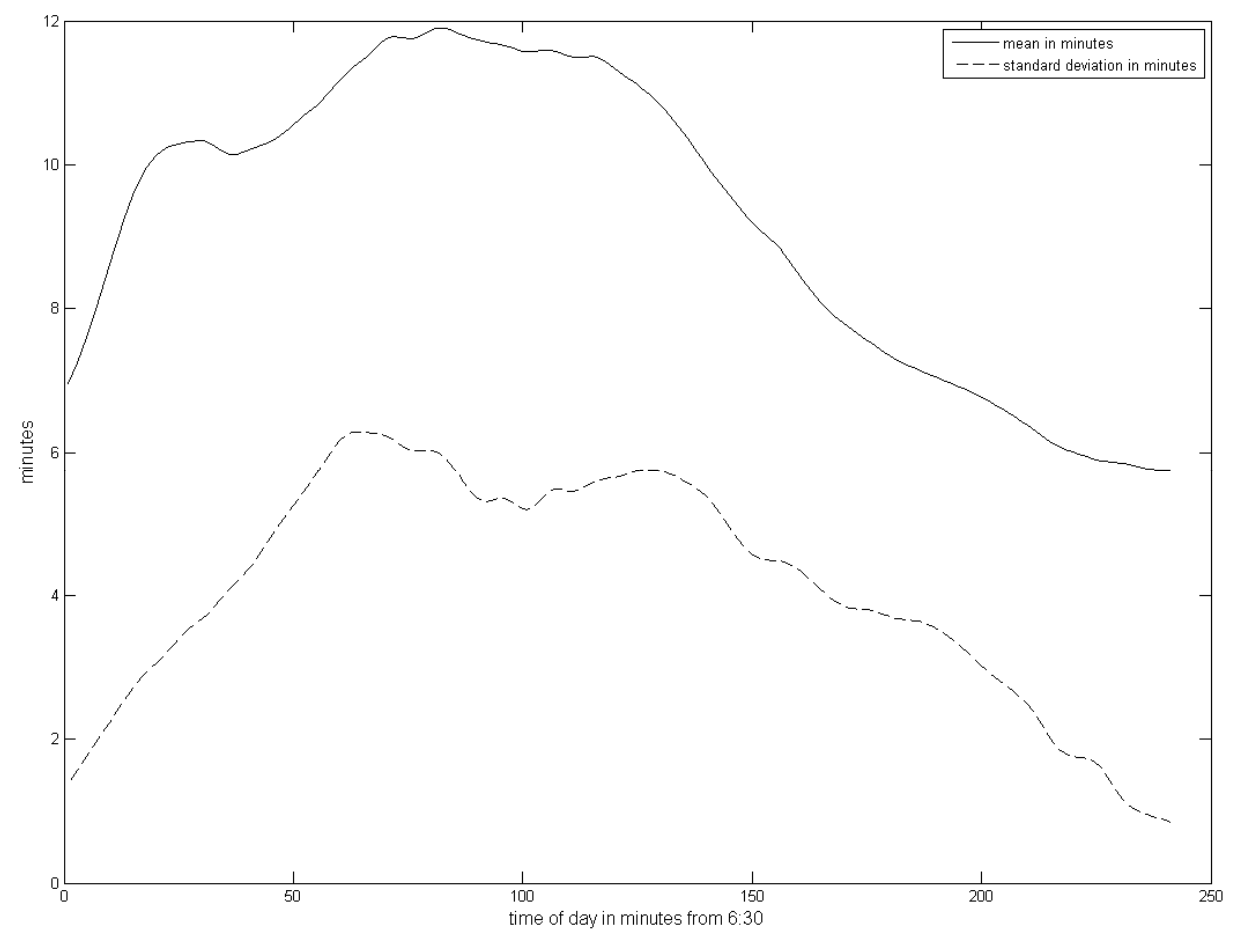


Figure 5: The effect of optimism and pessimism on the optimal $H$ for a time-of-day dependent travel time distribution $\left(t_{p a t}=8: 15\right)$.

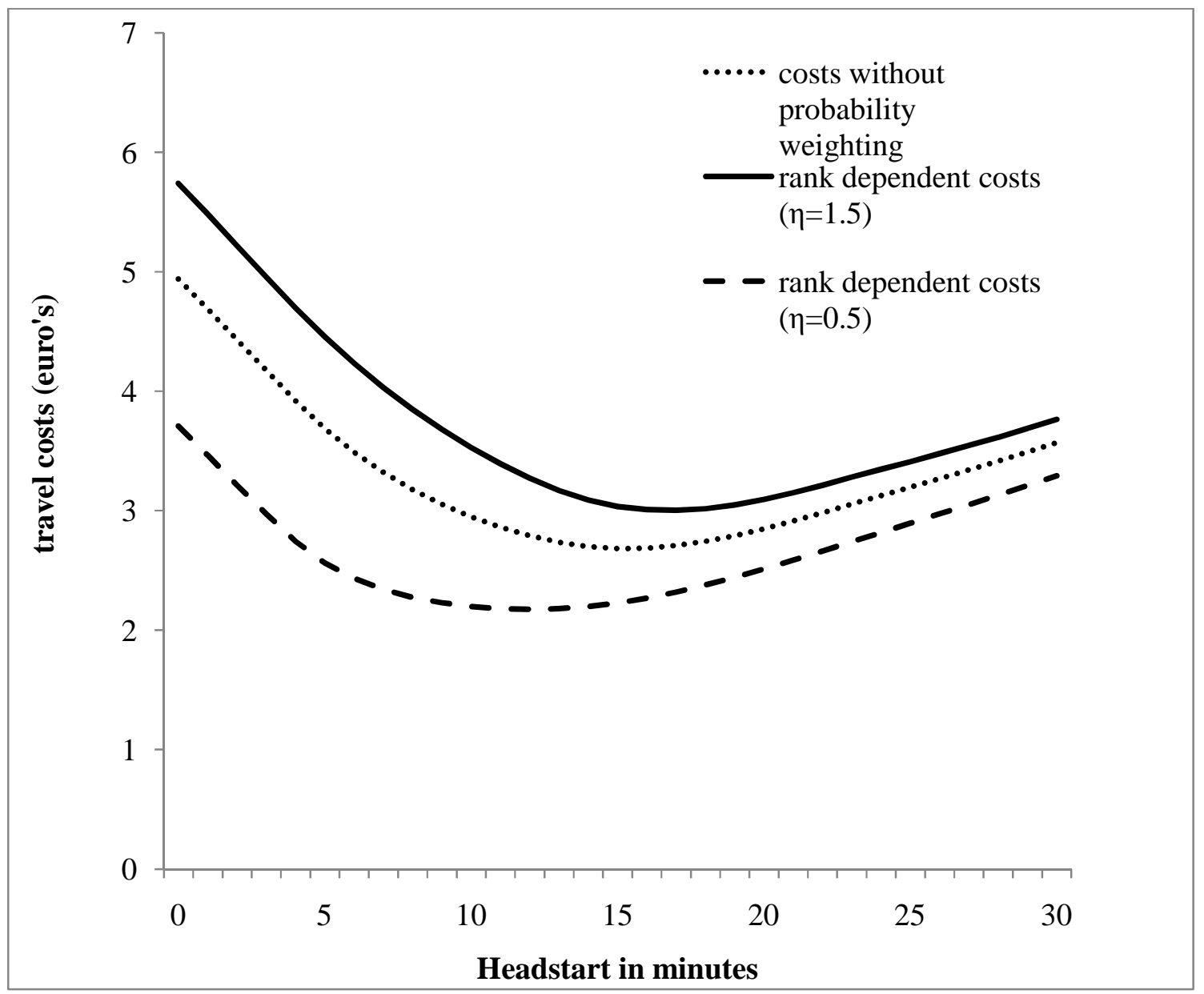


Figure 6: The $C O P W$ for a numerical example.

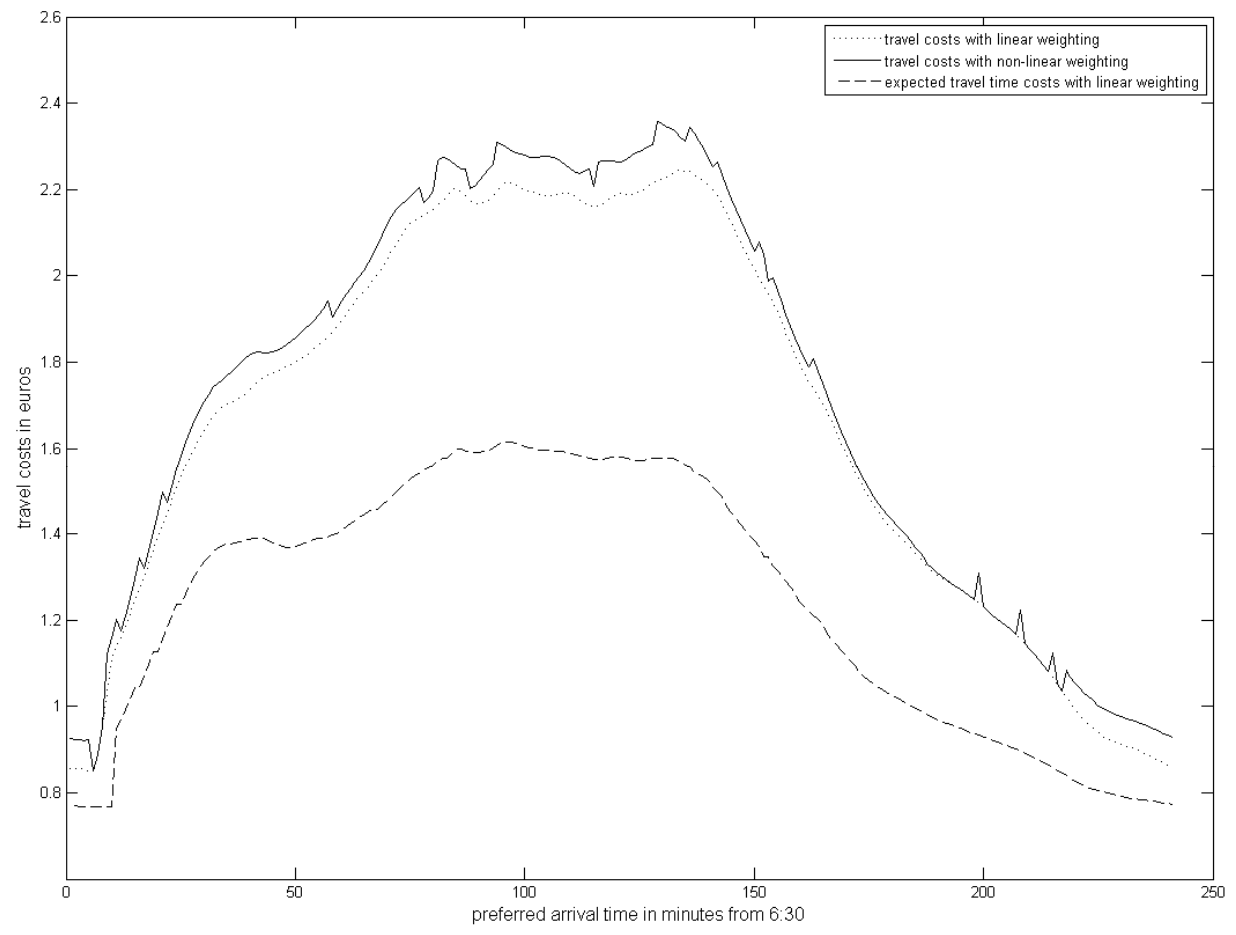


Figure 7: Average share of the total travel costs due to probability weighting

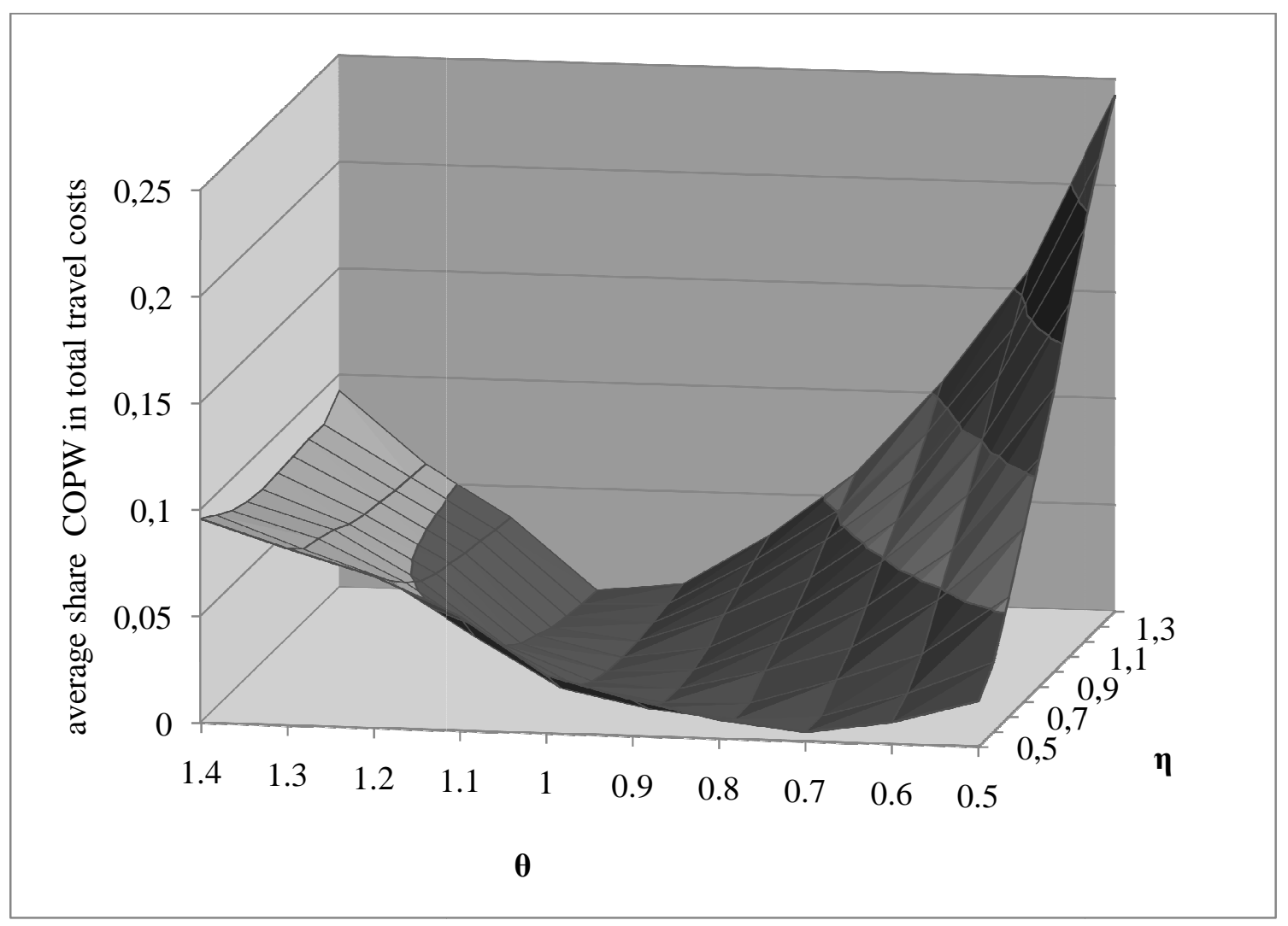


Table 1: Average percentage of the $C O P W$ in total travel costs for different WTP values.

\begin{tabular}{|l|llllll|}
\hline$\beta$ & $\alpha$ & $1.2 \alpha$ & $1.4 \alpha$ & $1.6 \alpha$ & $1.8 \alpha$ & $2 \alpha$ \\
\hline $0.2 \alpha$ & 1.6 & 1.7 & 1.8 & 1.9 & 2.0 & 2.1 \\
$0.4 \alpha$ & 2.3 & 1.9 & 2.3 & 2.4 & 2.4 & 2.6 \\
$0.6 \alpha$ & 2.9 & 3.1 & 3.1 & 3.0 & 2.7 & 2.9 \\
$0.8 \alpha$ & 2.8 & 3.4 & 3.7 & 3.7 & 3.9 & 3.8 \\
\hline
\end{tabular}

